\title{
Caractérisations physico-chimiques des eaux souterraines de la localité de Yamtenga (Burkina Faso)
}

\author{
Abdoulkadri AYOUBA MAHAMANE* et Boubié GUEL \\ Laboratoire de Chimie Moléculaire et des Matériaux, Equipe Chimie Physique et Electrochimie, \\ Département de Chimie, Université de Ouagadougou 03 BP 7021 Ouagadougou 03, Burkina Faso. \\ *Auteur correspondant ; E-mail: kadayouba@yahoo.fr, Tel : + 22678625451
}

\section{RESUME}

Dans la localité de Yamtenga au Burkina Faso, les eaux souterraines constituent les ressources hydriques primordiales pour l'alimentation en eau de boisson de la population rurale et pour l'irrigation des terres agricoles. Ce travail visait à étudier la qualité physico-chimique des eaux de forages et de puits de cette localité. Pour y parvenir, des prélèvements d'eau, au niveau de onze forages et puits, ont été effectués au cours de quatre séances de prélèvement à Yamtenga (Novembre 2010 à Octobre 2011). Différents paramètres physico-chimiques ont été mesurés : température, $\mathrm{pH}$, conductivité électrique, indice de Ryznar, turbidité, dureté. Les ions majeurs $\left(\mathrm{Na}^{+}, \mathrm{K}^{+}, \mathrm{Ca}^{2+}, \mathrm{Mg}^{2+}, \mathrm{HCO}_{3}^{-}, \mathrm{NO}_{3}{ }^{-}, \mathrm{SO}_{4}{ }^{2-}, \mathrm{Cl}^{-}\right)$ont été dosés par différentes méthodes dont la spectrométrie d'absorption atomique à flamme et la colorimétrie. Les éléments traces métalliques (ETMs) tels que : $\mathrm{Fe}^{2+}, \mathrm{Mn}^{2+}, \mathrm{Tl}^{+}, \mathrm{Cu}^{2+}$ et $\mathrm{Zn}^{2+}$ ont été dosés par des méthodes voltamétriques à redissolution. Les résultats ont montré que certains points d'eau ne sont pas conseillés à la consommation comme eau de boisson. Les paramètres qui déclassent ces eaux souterraines comme eau de boisson sont le fer, le manganèse et le thallium. Les eaux souterraines de Yamtenga sont classées bicarbonatées calciques et magnésiennes pour tous les ouvrages échantillonnés.

(C) 2015 International Formulae Group. All rights reserved.

Mots clés : Yamtenga, eaux souterraines, analyses physico-chimiques, éléments traces métalliques.

\section{INTRODUCTION}

Dans les pays en voie de développement à climat aride comme le Burkina Faso, le rôle des eaux souterraines est d'autant plus important qu'elles constituent souvent la seule source d'approvisionnement en eau potable et sont donc vitales pour le développement de ces pays (Bouchemal et al., 2011). La qualité naturelle des eaux souterraines peut être altérée par l'activité humaine ou par les divers éléments dont l'eau se charge au contact des terrains qu'elle rencontre. La détérioration de la qualité de l'eau est appréciée par les mesures des paramètres physico-chimiques. La qualité d'une eau est définie par des paramètres physiques, chimiques et biologiques, mais également par son usage. Ainsi, une eau impropre à la consommation peut être adaptée à l'irrigation ou à la pisciculture (Beauchamp, 2006).

Le présent travail s'intéresse à l'étude de la qualité physico-chimique des eaux de forages et de puits de Yamtenga, ainsi qu'à la 
détermination des principaux faciès chimiques. Il est important de connaître certains paramètres physico-chimiques, qui pourraient être la cause de nombreux problèmes car la mauvaise qualité de l'eau de boisson est un problème de santé publique. L'étude menée prend en compte les propriétés physico-chimiques et organoleptiques de cette localité.

\section{MATERIEL ET METHODES Présentation de la zone d'étude}

Yamtenga est un petit village situé au Sud-Est de la ville de Ouagadougou, de coordonnées géographiques en Universal Transverse Mercator (UTM) : X = 669126, $\mathrm{Y}=1363944$. Il appartient à la commune rurale de Bogodogo, qui est l'un des arrondissements de la commune de Ouagadougou. La localisation de la zone d'étude est indiquée sur la carte du Burkina Faso à la Figure 1.

La population de Yamtenga est estimée à 27300 habitants (Kombassere, 2007). La région est caractérisée par une longue saison sèche d'octobre à mai et une saison pluvieuse de juin à septembre. L'approvisionnement en eau se fait au niveau des forages et puits du village. La zone d'étude est relativement boisée avec de nombreux jardins et espaces de cultures où les habitants pratiquent les cultures de contre-saison. Ces pratiques sont rendues possibles grâce à un barrage d'eau et les nombreux puits et forages se trouvant dans le village.

\section{Prélèvement et conservation des échantillons}

Le prélèvement d'un échantillon d'eau est une opération assez délicate à laquelle le plus grand soin doit être apporté. Il conditionne les résultats analytiques et l'interprétation qui en sera donnée.
L'échantillon doit être homogène, représentatif et obtenu sans modifier les caractéristiques physico-chimiques de l'eau (Rodier, 2009). Il est donc nécessaire de développer une méthodologie adaptée à chaque cas et d'utiliser le matériel convenable. Quatre séances de prélèvements ont été réalisées selon le calendrier suivant: 06 novembre 2010, 06 février 2011, 30 juillet 2011 et 22 octobre 2011.

Les prélèvements ont été effectués au niveau de onze (11) points d'eau dont neuf (9) forages et deux (2) puits sur l'ensemble du village. Ces points d'eau ont été pour la plupart réalisés par des Organismes Non Gouvernementaux (ONG), par le biais de la coopération étrangère ou par l'Etat dans le cadre de l'hydraulique villageoise. Pour faciliter la manipulation des données, un numéro d'échantillon a été attribué à chaque point de prélèvement (Tableau 1).

Le paysage géologique de Yamtenga est constitué d'un granite gris à grain moyen, à biotite et épidote, renfermant localement d'abondantes enclaves sombres de quelques centimètres à quelques mètres de long et de deux classes granulométriques à grain moyen ou à grain fin.

La localisation des puits et forages dans la zone d'étude et la géologie de la zone d'étude sont indiquées sur la Figure 2.

Le matériel de prélèvement a fait l'objet d'une attention particulière. Ainsi, nous avons employé des flacons neufs en polyéthylène. Avant la sortie sur le terrain, les flacons sont d'abord lavés à grande eau, puis avec de l'acide nitrique $10 \%$ et finalement rincés abondamment avec de l'eau distillée. Sur le terrain avant remplissage des flacons, par mesure de sécurité, elles sont lavées trois (03) fois avec l'eau à analyser, puis remplis jusqu'au bord. Pour chaque point de prélèvement, deux flacons sont nécessaires. 
Le premier flacon est acidifié avec l'acide nitrique $(1 \mathrm{~mL} / \mathrm{L})$ et servira à l'analyse des éléments traces métalliques. Le $2^{\text {ème }}$ flacon non acidifié servira à l'analyse des paramètres physico-chimiques au laboratoire dans les heures qui suivent le prélèvement. Les échantillons prélevés, soigneusement étiquetés et conservé à $4{ }^{\circ} \mathrm{C}$ dans des glacières, sont transportés au laboratoire.

\section{Méthodologies et matériels utilisés pour les dosages}

Trois paramètres physico-chimiques (le $\mathrm{pH}$, la température et la conductivité électrique) ont été mesurés in-situ, immédiatement après le prélèvement de l'échantillon. Le $\mathrm{pH}$ est mesuré sur le terrain à l'aide d'un pH-mètre portable de marque Radiometer Analytical pHM201 et au laboratoire avec un pH-mètre de laboratoire de marque Hanna pH209. La prise de la température s'est effectuée à l'aide d'un thermomètre à affichage numérique. La mesure de la conductivité est faite sur le terrain grâce à un conductimètre portatif de marque Hanna. La turbidité est mesurée grâce à un turbidimètre, turbidity meterWAg-WT 3020. Son unité est la NTU (Nephelometric Turbidity Unit). Les ions sodium et potassium sont dosés par la spectrométrie d'absorption atomique à flamme (SAAF). L'appareil utilisé est un spectromètre d'absorption atomique de marque Varian AA 240FS. Les ions sulfates et nitrates sont déterminés par la spectrophotométrie ou colorimétrie à l'aide d'un spectrophotomètre de marque Hach Lange DR 3800. Les ions chlorures sont déterminés par la méthode de Mohr. Les ions calcium et magnésium sont déterminés par complexométrie à l'aide de l'EDTA. Le Titre Alcalimétrique Complet (TAC) est déterminé par volumétrie. L'indice de Ryznar (IRz) est déterminé par la méthode de mesure du pH de saturation.

Le dosage des Eléments Traces Métalliques (ETMs) fer (II), manganèse (II), thallium (I), cuivre (II) et zinc (II) a été réalisé par les méthodes électrochimiques, à savoir les méthodes voltamétriques à redissolution. Le fer (II) a été déterminé par la voltamétrie à redissolution cathodique à l'aide d'une électrode à pâte de carbone chimiquement modifiée (EPCCM) par la 1,10-phénantroline et le Nafion. Le manganèse (II) a été déterminé par la voltamétrie à redissolution cathodique d'un complexe adsorbé sur une électrode à pâte de carbone. Comme agent complexant, nous avons utilisé le 2-(5'Bromo-2'-pyridylazo)-5-diethylaminophénol (5-Br-PADAP). La détermination du thallium, du zinc et du cuivre a été effectuée par la voltamétrie à redissolution anodique sur film de mercure.

Pour l'évaluation de la qualité physicochimique des eaux souterraines étudiées, les valeurs guides de l'OMS (Organisation Mondiale de la Santé) des paramètres physico-chimiques pour la qualité eau de boisson (UNE, 2007) et la norme américaine (OEHHA, 1999) pour le thallium ont été utilisées.

\section{Bilans ioniques}

La balance ionique (BI) s'appuie sur le principe que la somme des anions majeurs et la somme des cations majeurs sont équivalentes (concentrations exprimées en méq/L) :

$[\mathrm{Cl}]+\left[\mathrm{SO}_{4}{ }^{2-}\right]+\left[\mathrm{NO}_{3}^{-}\right]+\left[\mathrm{HCO}_{3}^{-}\right]=\left[\mathrm{Ca}^{2+}\right]+$ $\left[\mathrm{Mg}^{2+}\right]+\left[\mathrm{Na}^{+}\right]+\left[\mathrm{K}^{+}\right]$

L'erreur en \% est donnée par :

$\mathrm{BI}=\frac{\sum_{\text {cations }}-\sum_{\text {anions }}}{\sum_{\text {cations }}+\sum_{\text {anions }}} \times 100$ 
Une analyse chimique des eaux n'est considérée comme représentative et acceptable que lorsque la balance ionique est inférieure ou égale à $10 \%$ (Kouassi et al., 2013).

\section{Faciès hydrochimiques}

Dans le but de bien identifier les faciès hydrochimiques et d'avoir une indication sur l'aspect qualitatif des eaux souterraines, la représentation graphique des résultats d'analyses s'avère un outil inévitable. Pour atteindre cet objectif, on a eu recours au diagramme de Piper. La réalisation de ces diagrammes a été faite en utilisant le logiciel Diagrammes 5.6, conçu par Simler (2007). Le diagramme de Piper permet de représenter le faciès chimique d'un ensemble d'échantillons d'eaux. Il est composé de deux triangles permettant de représenter le faciès cationique et le faciès anionique et d'un losange synthétisant le faciès global. Les nuages de points concentrés dans un pôle représentent pour les différents échantillons la combinaison des éléments cationiques et anioniques. Ce type de diagramme est particulièrement adapté pour étudier l'évolution du faciès des eaux lorsque la minéralisation augmente ou bien pour indiquer les types de cations et anions dominants et pour distinguer des groupes d'échantillons.

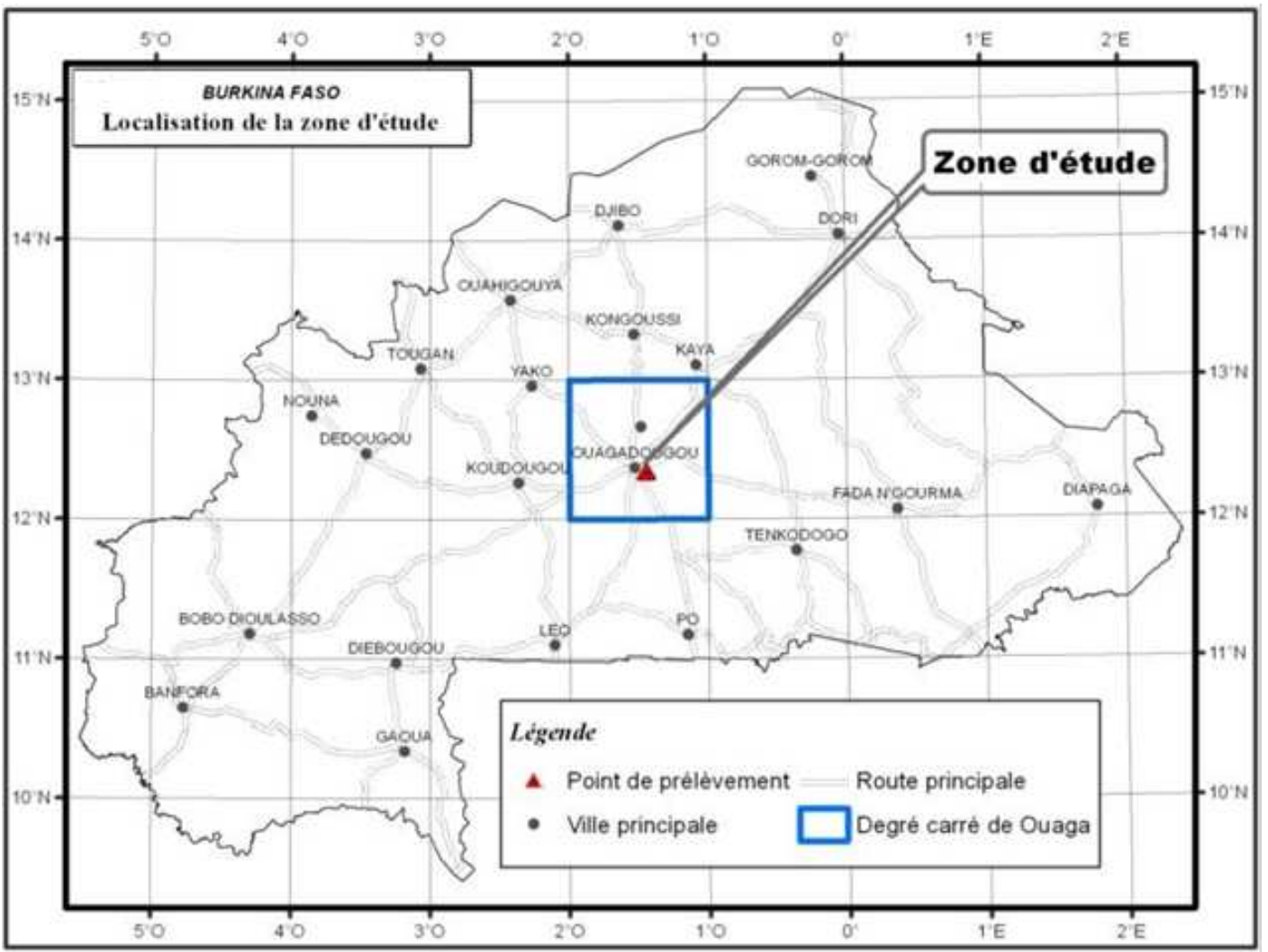

Figure 1: Localisation de la zone d'étude Yamtenga sur la carte du Burkina Faso. 


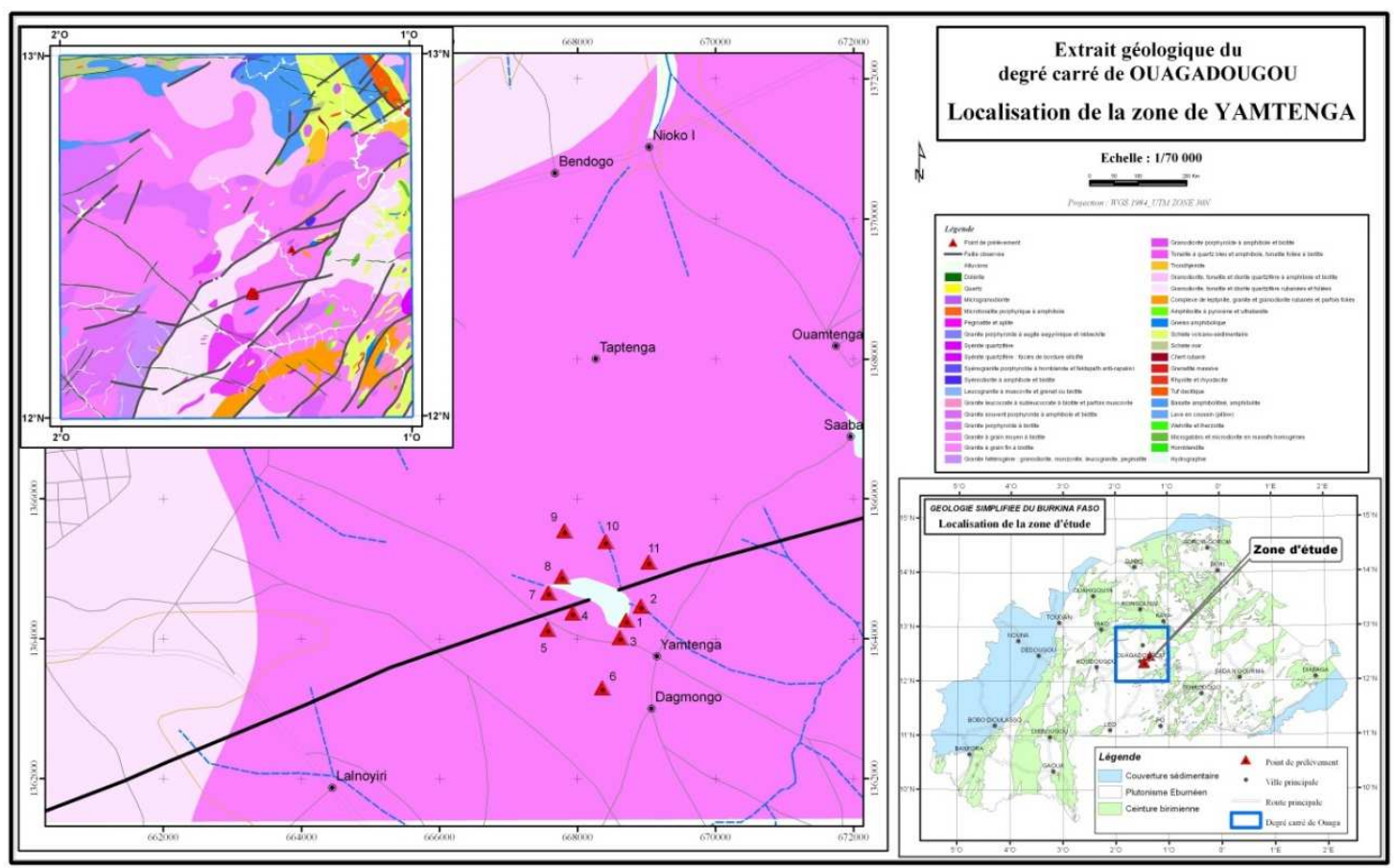

Figure 2: Localisation des puits et forages dans la zone d'étude.

Tableau 1: Numérotation des lieux de prélèvement.

\begin{tabular}{llll}
\hline $\mathbf{N}^{\circ}$ d'échantillon & Description du point d'eau & $\begin{array}{l}\text { Coordonnées } \\
\text { (UTM) }\end{array}$ & géographiques \\
\cline { 3 - 4 } & & $\mathbf{X}$ & $\mathbf{Y}$ \\
\hline $\mathbf{1}$ & Forage au sein de l'école primaire de Yamtenga & 668608 & 1364016 \\
$\mathbf{2}$ & Puits au sein des jardins potagers & 668700 & 1364264 \\
$\mathbf{3}$ & Forage à côté du CSPS (centre de santé) & 668914 & 1364466 \\
$\mathbf{4}$ & Forage sur la route du marché & 663124 & 1389698 \\
$\mathbf{5}$ & Forage dit pompe de Daniell & 667573 & 1364658 \\
$\mathbf{6}$ & Forage derrière le lycée municipal & 667562 & 1364135 \\
$\mathbf{7}$ & Forage dans la cour de la mosquée & 667770 & 1364890 \\
$\mathbf{8}$ & Puits à côté duquel les femmes lavent le linge & 667926 & 1364370 \\
$\mathbf{9}$ & Forage devant la maison du chef de village & 667804 & 1365541 \\
$\mathbf{1 0}$ & Forage au niveau de l'école privée le Nazaréen & 668403 & 1365389 \\
$\mathbf{1 1}$ & Forage dit robinet Saint Camille & 669023 & 1365095 \\
\hline UTM: Universal Transverse Mercator. & &
\end{tabular}




\section{RESULTATS \\ La température}

Nous avons relevé (Figure 3) des valeurs de températures relativement basses pendant la première séance d'échantillonnage qui s'est déroulée en novembre, période correspondant au début de la saison sèche au Burkina Faso et ces températures ne présentent pas de grandes variations d'un point à un autre. La valeur minimale est de 17 ${ }^{\circ} \mathrm{C}$ et la valeur maximale de $20,6{ }^{\circ} \mathrm{C}$ pour le premier échantillonnage. Au cours des autres séries d'échantillonnage, les valeurs de température sont plus élevées, mais on note que les températures varient également peu d'un point à l'autre. Pour la $2^{\mathrm{ème}}$ série faite en février, la valeur minimale est de $27{ }^{\circ} \mathrm{C}$ et la maximale $30,5^{\circ} \mathrm{C}$. Au cours de la $3^{\text {ème }}$ série en juillet, nous avons relevé des températures allant de 30 à $34{ }^{\circ} \mathrm{C}$. Enfin, au cours de la dernière série en octobre, la valeur minimale relevée est de $29,8{ }^{\circ} \mathrm{C}$ et la maximale de 33,8 ${ }^{\circ} \mathrm{C}$.

\section{Le pH}

La plupart des échantillons ont un $\mathrm{pH}<$ 7 excepté quelques échantillons de la $4^{\text {ème }}$ séance de prélèvement. Nos échantillons d'eau (Figure 4) sont acides et se situent presque tous dans la plage de $\mathrm{pH}$ recommandé par l'OMS (6,5-8), exceptés quelques-uns qui ont un $\mathrm{pH}<$ à 6,5 .

\section{La conductivité électrique}

La mesure de la conductivité électrique permet d'évaluer rapidement, mais très approximativement la minéralisation globale de l'eau et d'en suivre l'évolution. Les valeurs de conductivité électrique obtenues varient entre $118 \mu \mathrm{S} / \mathrm{cm}$ et $500 \mu \mathrm{S} / \mathrm{cm}$ (Figure 5). Sur les 4 séances d'échantillonnage, il est à noter que tous les points d'eau à l'exception du point 10 sont faiblement minéralisés. L'échantillon 10 présente des valeurs plus élevées de conductivité que les autres échantillons, sa conductivité est supérieure à la norme OMS qui est de $300 \mu \mathrm{S} / \mathrm{cm}$. Cette eau est moyennement minéralisée.

\section{L'indice de Ryznar}

Toutes les eaux analysées ont un indice de Ryznar supérieur à 8,5 comme on le voit sur l'histogramme de la Figure 6. Ces valeurs révèlent un caractère corrosif de ces eaux.

\section{La turbidité}

D'après la Figure 7, seuls les points 2 et 8 présentent des valeurs de turbidité dépassant la norme OMS qui est de 5 NTU.

\section{La dureté}

La dureté totale d'une eau est produite par les sels de calcium et de magnésium qu'elle contient. La dureté de l'eau influe essentiellement sur l'état des canalisations et des appareils de chauffage, et sur le lavage du linge. Tous les échantillons d'eau présentent de faibles valeurs de dureté et sont des eaux douces, excepté l'échantillon 10 (Figure 8).

\section{Le calcium}

Le calcium est un métal alcalinoterreux extrêmement répandu dans la nature et en particulier dans les roches calcaires sous forme de carbonates. C'est le composant majeur de la dureté de l'eau. Dans nos échantillons d'eau, les teneurs en calcium varient de $8,8 \mathrm{mg} / \mathrm{L}$ à $60,8 \mathrm{mg} / \mathrm{L}$ (Figure 9). Aucune de ces eaux ne présente donc de concentration supérieure à la norme OMS qui est de $100 \mathrm{mg} / \mathrm{L}$.

\section{Le magnésium}

Le magnésium constitue le deuxième élément significatif de la dureté de l'eau après le calcium. Il est présent dans les eaux de cette région à des teneurs allant de 0,48 à 26,16 $\mathrm{mg} / \mathrm{L}$ (Figure 10). Ces teneurs sont inférieures la norme admise par l'OMS pour le magnésium, qui est de $150 \mathrm{mg} / \mathrm{L}$. 


\section{Le sodium}

L'analyse en ions sodium des échantillons d'eau sur l'ensemble des 4 séries d'échantillonnage nous donne des teneurs allant de 2,93 à 22,32 mg/L (Figure 11), ce qui est sans danger pour la santé, la valeur limite recommandée par l'OMS étant de 200 $\mathrm{mg} / \mathrm{L}$.

\section{Le potassium}

Les valeurs en potassium que nous avons mesurées par la spectrométrie d'absorption atomique à flamme (Figure 12) varient de 0,95 à $12,88 \mathrm{mg} / \mathrm{L}$. Elles sont bien en deçà de la norme OMS (200mg/L).

\section{Les bicarbonates}

A l'aide des valeurs de Titre Alcalimétrique Complet (TAC) mesurées, nous avons déterminé la teneur en bicarbonates dans les échantillons d'eau (Figure 13). Il n'y a pas de norme OMS pour cet élément, mais une concentration élevée en bicarbonates donne une saveur salée à l'eau. Les teneurs varient de 39,04 à 268,4 mg/L. Ils sont particulièrement élevés au point 10 avec une valeur maximale de 268,4 mg/L.

\section{Les nitrates}

$\mathrm{Au}$ niveau de la région étudiée, les teneurs en nitrates varient durant la période d'étude (Figure 14) de $0,2 \mathrm{mg} / \mathrm{L}$ (point 4) à $2,8 \mathrm{mg} / \mathrm{L}$ (point 8). Ces valeurs restent inférieures à la valeur admissible par la norme OMS qui est de $50 \mathrm{mg} / \mathrm{L}$.

\section{Les sulfates}

Les valeurs de ce paramètre dans les eaux étudiées sont très variables comme on peut le voir sur la Figure 15. Elles oscillent entre 0 et $9 \mathrm{mg} / \mathrm{L}$, valeurs en dessous des normes OMS (250 mg/L). Les valeurs élevées en sulfates ont été mesurés au niveau des points 2 et 8 qui sont des puits.

\section{Les chlorures}

D'après la Figure 16, les teneurs en chlorure varient entre 0 et $35 \mathrm{mg} / \mathrm{L}$, ce qui est sans danger pour une consommation des eaux car la norme OMS est de $250 \mathrm{mg} / \mathrm{L}$. Comme dans le cas des sulfates, les pics ont été enregistrés aux points d'eau 2 et 8 qui sont des puits.

\section{Le fer}

Les concentrations en fer (II) comme on peut le voir sur la Figure 17, varient de 0 à 2,06 mg/L. La quasi-totalité des eaux ont des teneurs dépassant la norme OMS qui est de $0,3 \mathrm{mg} / \mathrm{L}$.

\section{Le manganèse}

Le manganèse accompagne généralement le fer dans les roches et dans les eaux. Naturellement, les concentrations en manganèse des eaux souterraines sont en moyenne 10 fois moins élevées que les concentrations en fer. Les teneurs en manganèse des eaux analysées varient de 0,004 à $1,738 \mathrm{mg} / \mathrm{L}$ comme on peut le voir sur la Figure 18. Ces teneurs sont variables d'un point d'eau à l'autre et aussi en fonction de la période de prélèvement. Les eaux présentant des concentrations supérieures à la norme OMS $(0,4 \mathrm{mg} / \mathrm{L})$ sont les points $2,4,5,8$ et 10 .

\section{Le thallium}

Le thallium est un métal lourd extrêmement dangereux. Les teneurs en thallium de tous les échantillons d'eaux sont regroupées dans la Figure 19. Les teneurs varient de $0,0732 \mu \mathrm{g} / \mathrm{L}$ à $3,94 \mu \mathrm{g} / \mathrm{L}$. Les pics de concentration dépassant la norme américaine recommandée par Office of Environmental Health Hazard Assessment (OEHHA) qui est de $2 \mu \mathrm{g} / \mathrm{L}$, ont été enregistrés aux points $2,5,8$ et 11 . Ces eaux sont donc déconseillées pour une utilisation comme eau de boisson.

\section{Le zinc}

La Figure 20 regroupe les teneurs en zinc de tous nos échantillons d'eau analysés. Les teneurs varient de 0 à $4,18 \mathrm{mg} / \mathrm{L}$. Exceptés les points 7 et 9 où nous avons des teneurs respectivement de 4,18 et $3,28 \mathrm{mg} / \mathrm{L}$, tous les autres points d'eau présentent des 
teneurs inférieures à la norme OMS qui est de $3 \mathrm{mg} / \mathrm{L}$. La présence dans ces eaux de cet élément métallique est donc sans danger.

\section{Le cuivre}

Toutes les eaux analysées (Figure 21) présentent de faibles teneurs en cuivre inférieures à la norme OMS qui est $2 \mathrm{mg} / \mathrm{L}$, excepté le point 6 où une concentration élevée de $5,14 \mathrm{mg} / \mathrm{L}$ a été mesurée (cette concentration n'est pas représentée sur l'histogramme). Cette teneur élevée pourrait être la résultante d'une corrosion de la tuyauterie ou une pollution industrielle.

\section{Faciès hydrochimiques}

Les diagrammes de piper correspondant aux échantillons d'eau des quatre séances de prélèvement sont représentés à la Figure 22.

Sur les Figures 22a et 22b, on observe dans le triangle des anions une prédominance des bicarbonates, ce qui traduit un faciès bicarbonaté. Dans le triangle des cations, il n'y a pas d'ions dominant, ce qui traduit un faciès mixte sodique, potassique, calcique et magnésien. Ces résultats sont confirmés dans le losange où nous avons un faciès global bicarbonaté calcique et magnésien.

Sur les diagrammes de piper représentés par les Figures 22c et 22d, on note toujours le faciès bicarbonaté pour les anions. Pour les cations nous avons un faciès mixte calcique, sodique et magnésien avec une faible dominance pour le calcique. Le losange symbolisant le faciès global nous montre un faciès bicarbonaté calcique et magnésien. On note également un début d'évolution du pôle bicarbonaté vers le pôle chlorures + nitrates traduisant une certaine empreinte anthropique.

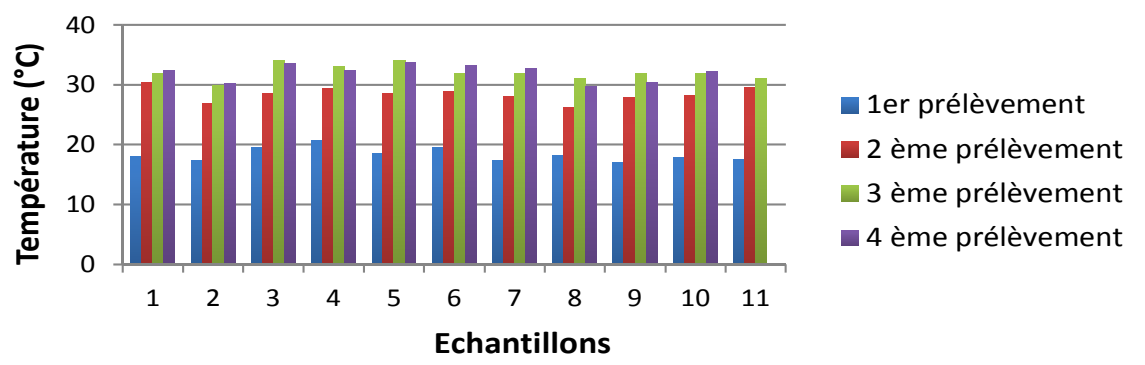

Figure 3: Evolution spatio-temporelle de la Température $\left({ }^{\circ} \mathrm{C}\right)$.

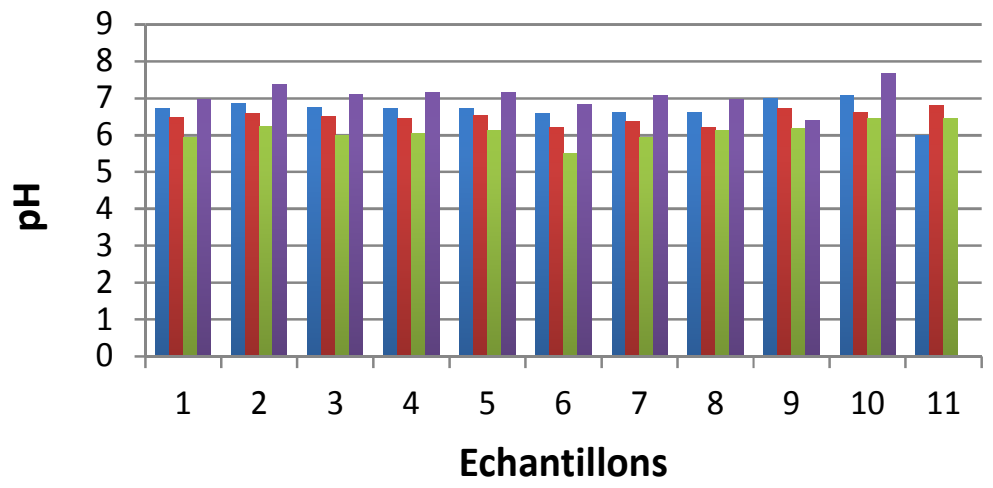

1er prélèvement

2 ème prélèvement

- 3 ème prélèvement

4 ème prélèvement

Figure 4: Evolution spatio-temporelle du $\mathrm{pH}$. 


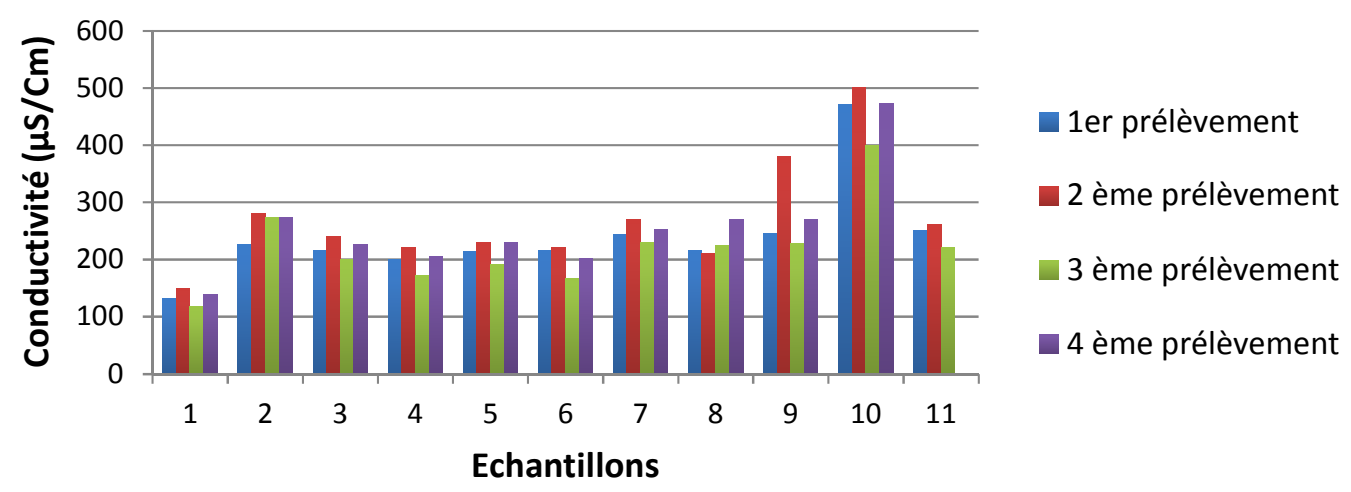

Figure 5: Evolution spatio-temporelle de la conductivité électrique $(\mu \mathrm{S} / \mathrm{cm})$.

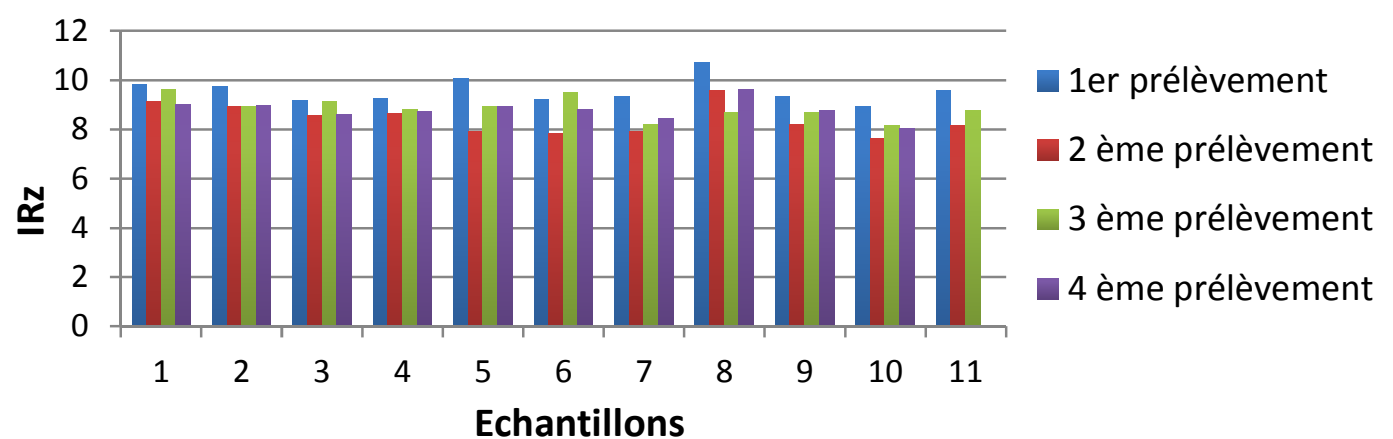

Figure 6: Evolution spatio-temporelle de l'indice de Ryznar (IRz).

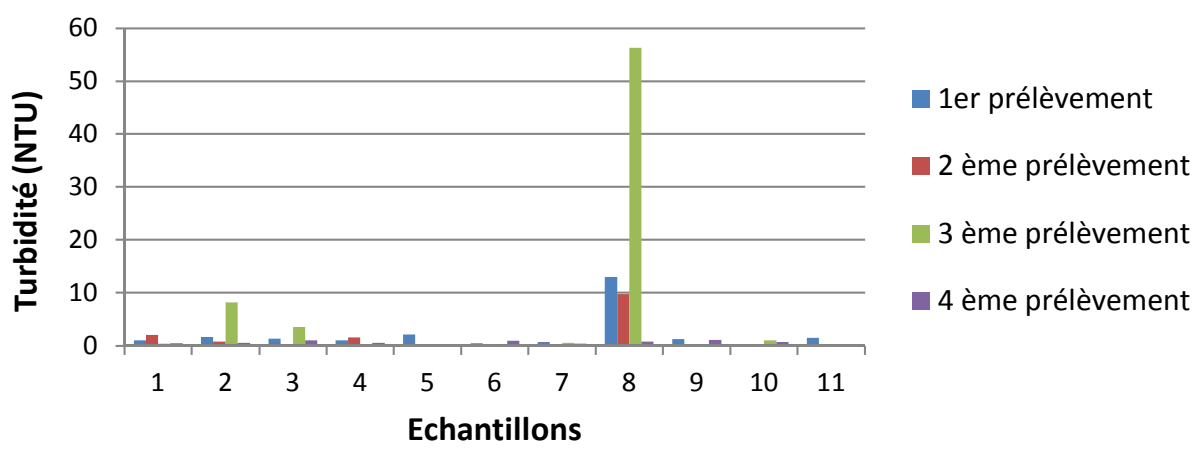

Figure 7: Evolution spatio-temporelle de la turbidité. 


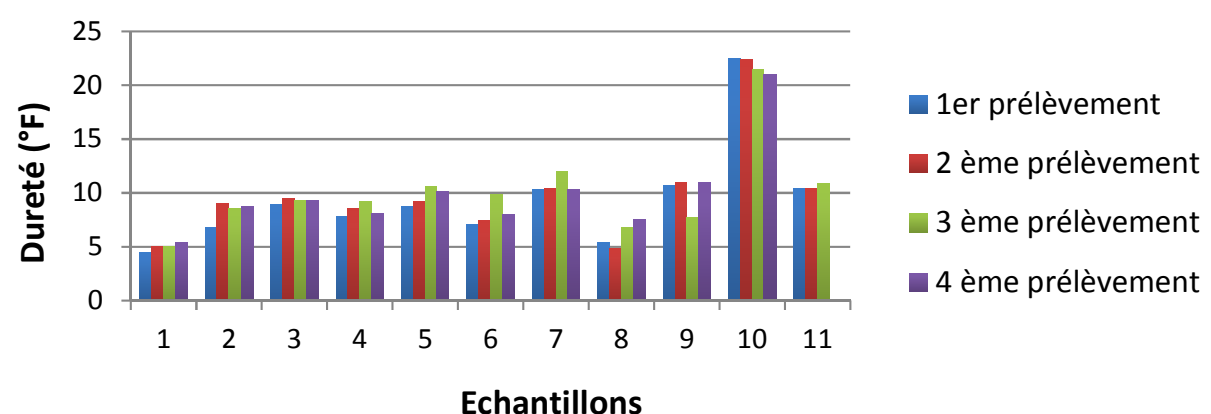

Figure 8: Evolution spatio-temporelle de la dureté.

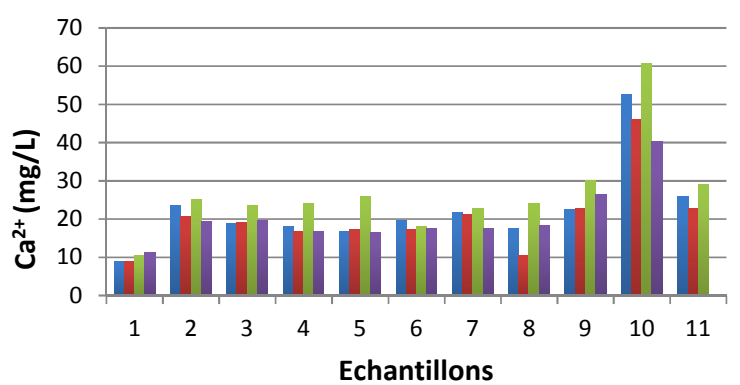

- 1er prélèvement

- 2 ème prélèvement

- 3 ème prélèvement

- 4 ème prélèvement

Figure 9: Evolution spatio-temporelle de la teneur en calcium.

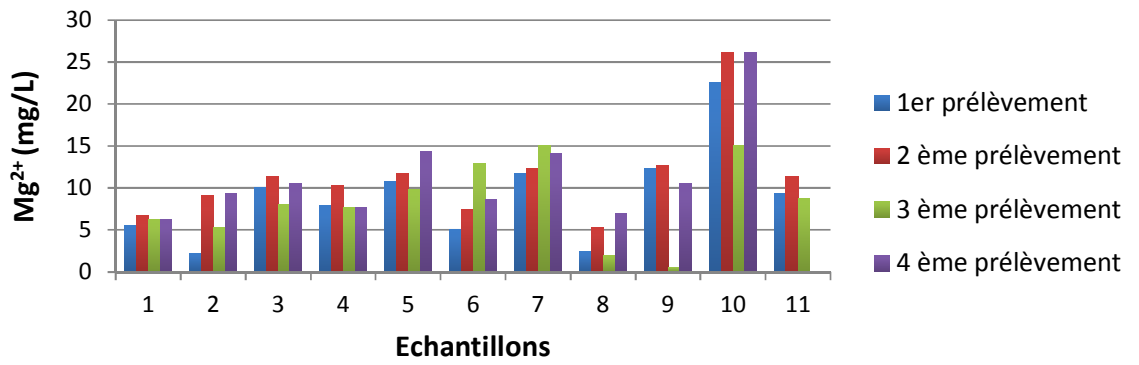

Figure 10: Evolution spatio-temporelle de la teneur en magnésium.

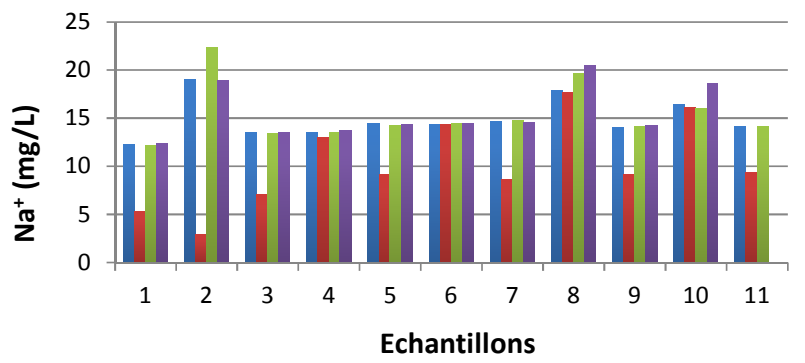

匹 1er prélèvement

च 2 ème prélèvement

■ 3 ème prélèvement

口 4 ème prélèvement

Figure 10: Evolution spatio-temporelle de la teneur en sodium. 


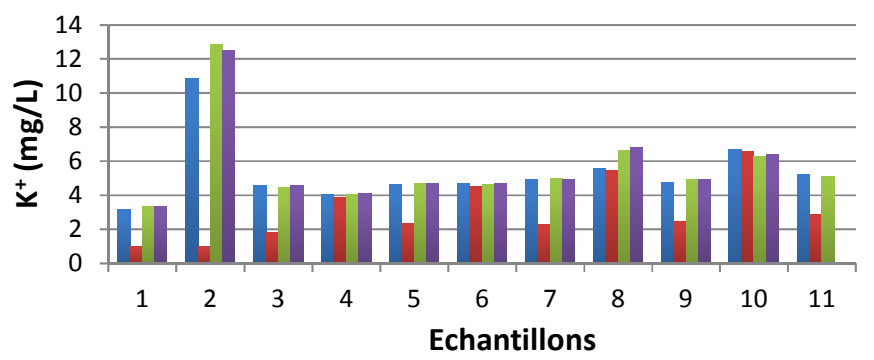

1er prélèvement

- 2 ème prélèvement

- 3 ème prélèvement

4 ème prélèvement

Figure 12: Evolution spatio-temporelle de la teneur en potassium.

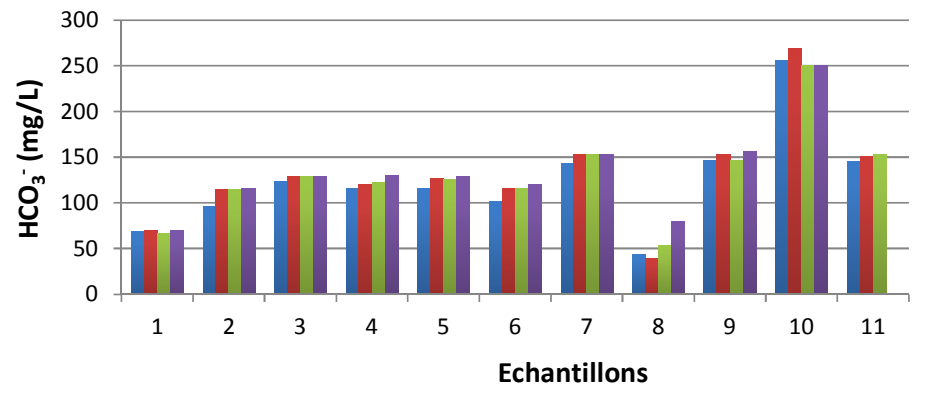

1er prélèvement

2 ème prélèvement

3 ème prélèvement

4 ème prélèvement

Figure 13: Evolution spatio-temporelle de la teneur en ions bicarbonates.

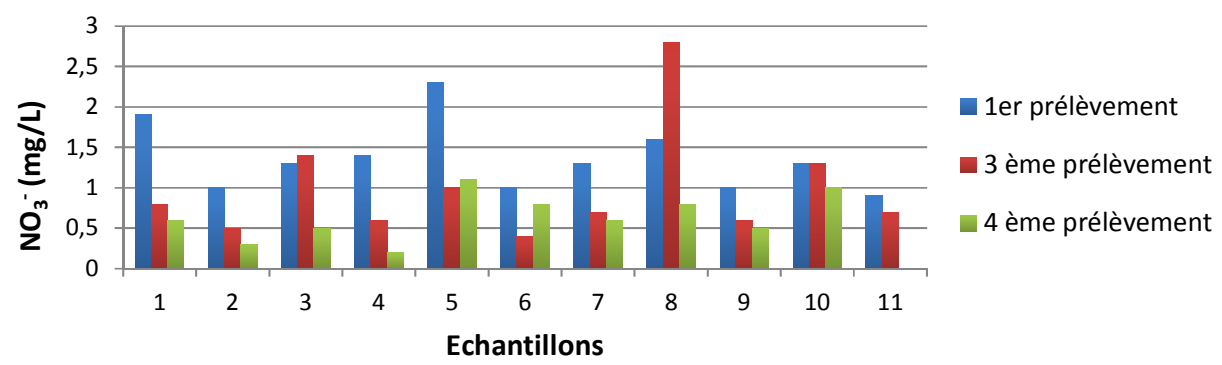

Figure 14: Evolution spatio-temporelle de la teneur en nitrates.

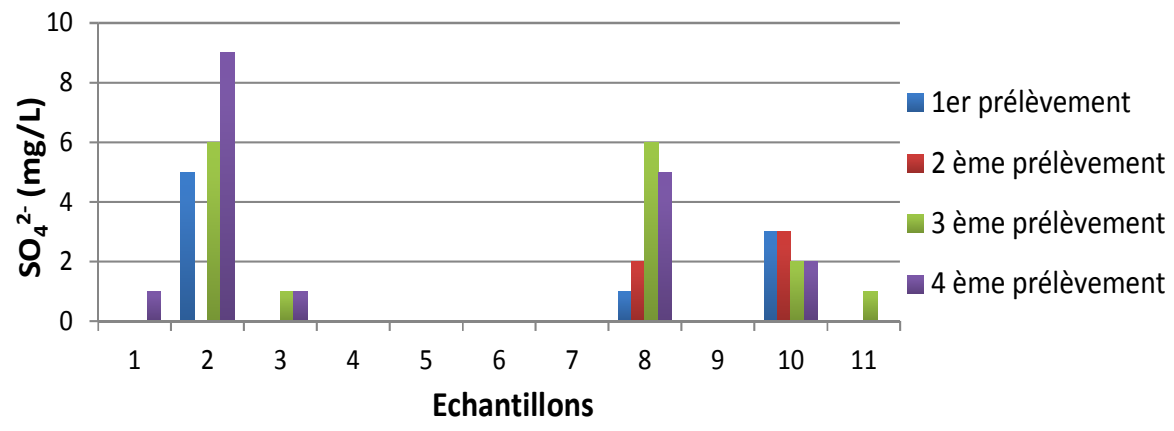

Figure 15: Evolution spatio-temporelle de la teneur en sulfates. 


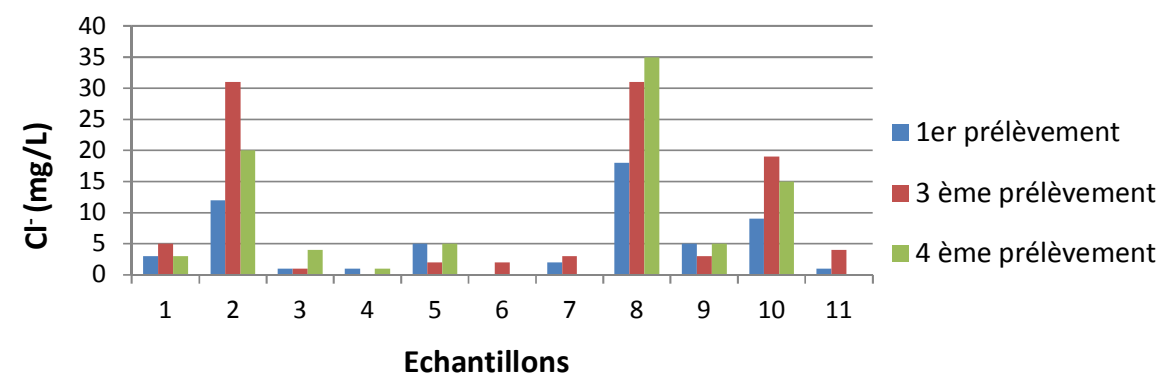

Figure 16: Evolution spatio-temporelle de la teneur en chlorures.

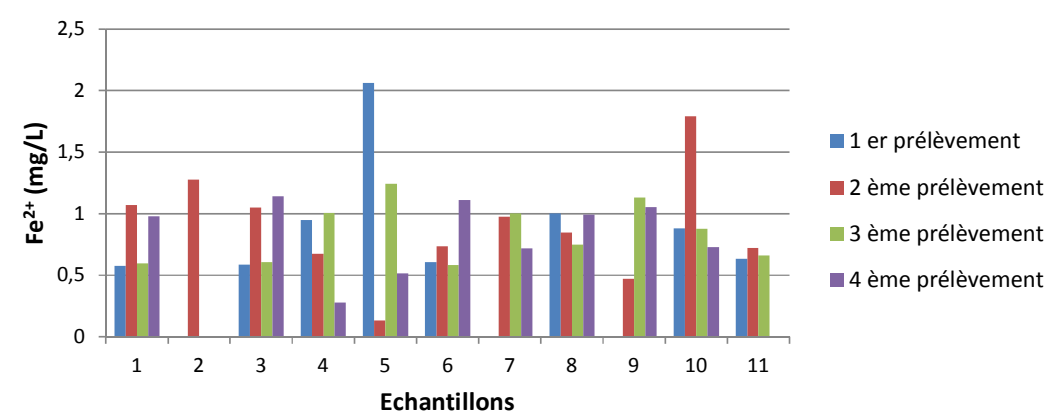

Figure 17: Evolution spatio-temporelle de la teneur en fer (II).

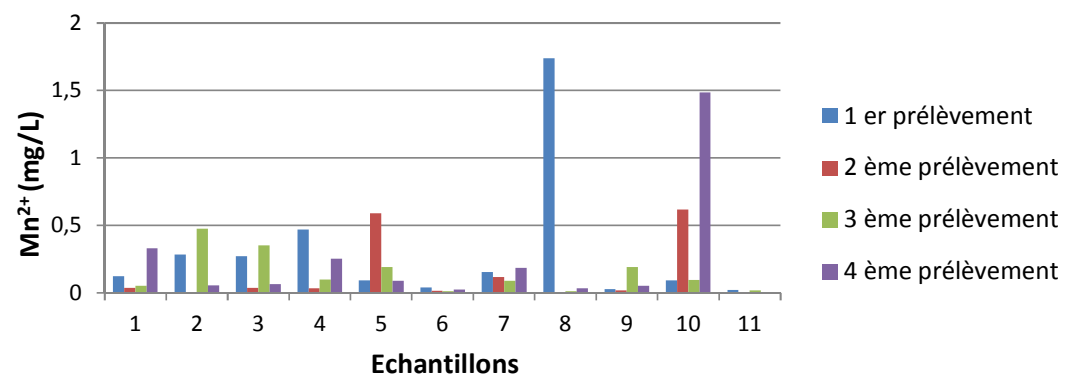

Figure 18: Evolution spatio-temporelle de la teneur en manganèse (II).

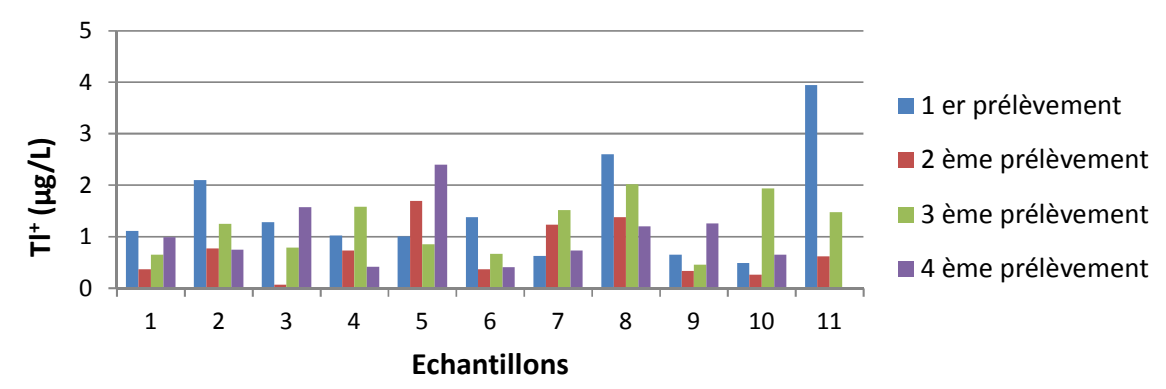

Figure 19: Evolution spatio-temporelle de la teneur en thallium 


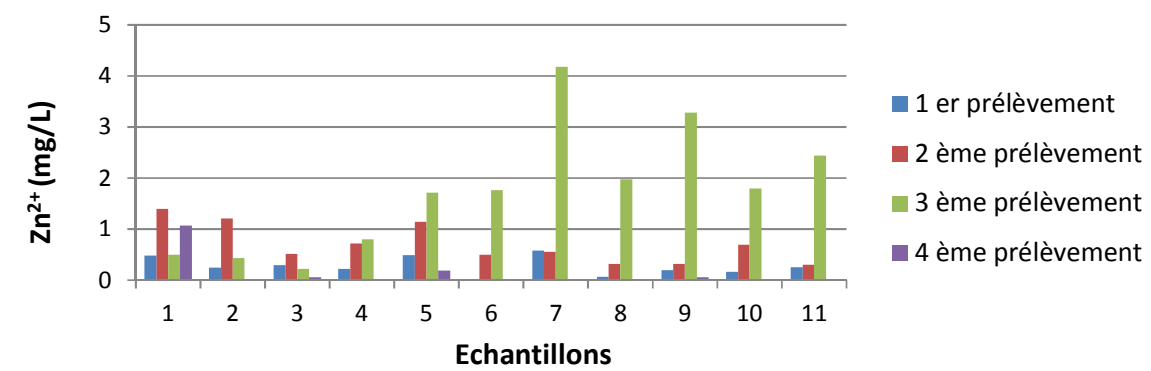

Figure 20 : Evolution spatio-temporelle de la teneur en zinc.

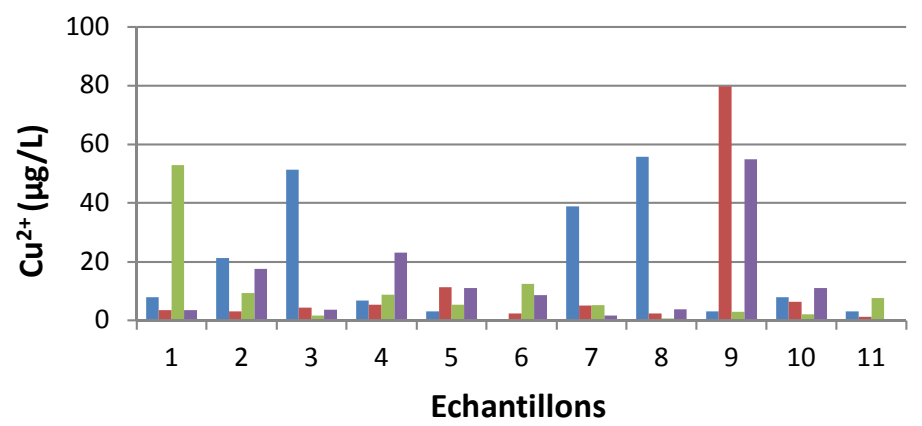

1 er prélèvement

2 ème prélèvement

3 ème prélèvement

- 4 ème prélèvement

Figure 21: Evolution spatio-temporelle de la teneur en cuivre. (1 ${ }^{\text {ère }}$ Campagne, l'échantillon $6: 5140 \mu \mathrm{g} / \mathrm{L}$ n'est pas représenté sur le graphique).

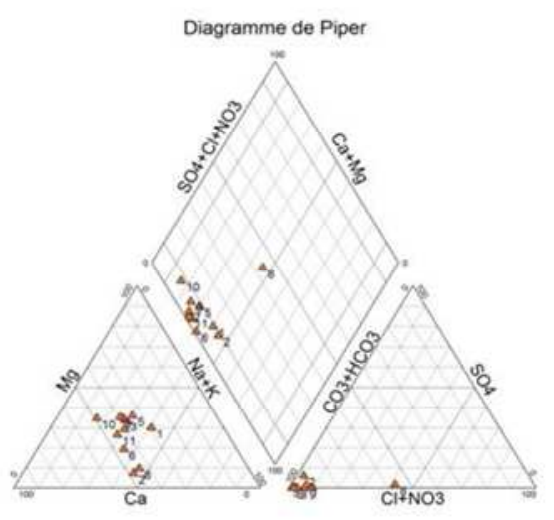

(a)

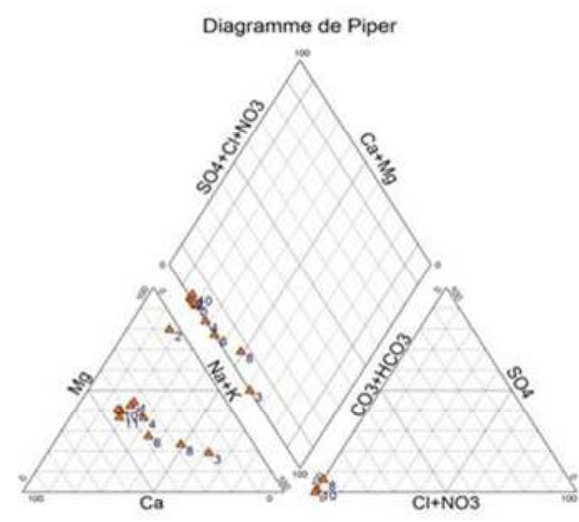

(b) 


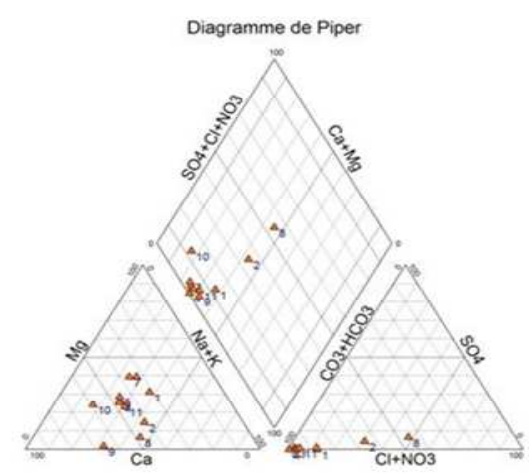

(c)

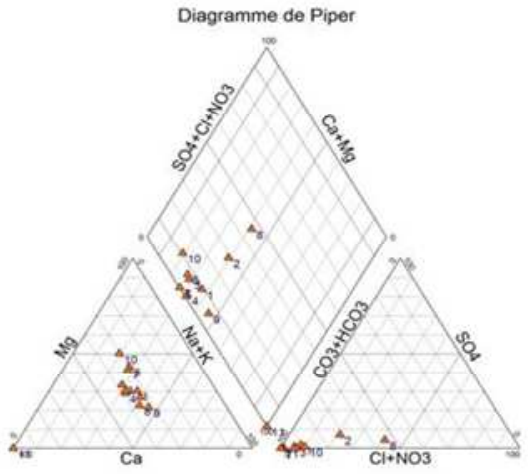

(d)

Figure 22: Diagramme de piper des séances de prélèvement. (a) : $1^{\text {ère }}$ séance de prélèvement, (b) : $2^{\text {ème }}$ séance de prélèvement, (c) : $3^{\text {ème }}$ séance de prélèvement, (d) $: 4^{\text {ème }}$ séance de prélèvement.

\section{DISCUSSION}

Les valeurs de températures relevées sont en accord avec ce qui est rapporté dans la littérature : les eaux souterraines sont moins sensibles aux variations de température que les eaux superficielles (Rodier, 2009). Les valeurs de $\mathrm{pH}$ sont conformes à la norme OMS, exceptés des échantillons qui sont acides. Selon Rodier (2009), ces eaux ne sont pas à priori interdites pour la consommation humaine, mais cette acidité leur confère une propriété corrosive plus importante du ciment ou des métaux de canalisation. Un contact prolongé, notamment la stagnation de l'eau dans les tuyaux en acier pendant les pauses de la nuit engendre le phénomène de corrosion selon la réaction $(\mathrm{LH} / \mathrm{UO}, 2004)$ :

$$
\mathrm{Fe}+2 \mathrm{H}_{3} \mathrm{O}^{+} \rightarrow \mathrm{Fe}^{2+}+2 \mathrm{H}_{2} \mathrm{O}+\mathrm{H}_{2}
$$

La présence des ions $\mathrm{Fe}^{2+}$ engendrés par ce mécanisme confère la coloration rougeâtre à l'eau observée dans les premières heures de pompage dans la matinée. D'une manière générale, les valeurs de $\mathrm{pH}$ obtenues pour ces eaux souterraines sont conformes aux résultats obtenus par d'autres études publiées dans la littérature car dans la plupart des eaux souterraines, le pH est compris entre 6 et 8,5 (El Asslouj et al., 2007; Matini et al., 2009; Derwich et al., 2010; Belghiti et al., 2013).
Les eaux de Yamtenga sont faiblement minéralisées. Ces eaux faiblement minéralisées sont convenables pour la consommation. Ouandaogo/Yaméogo (2008), qui a étudié la conductivité électrique des eaux de certains forages à Ouagadougou, a trouvé des valeurs de conductivités électriques variant de 150 à $444 \mu \mathrm{S} / \mathrm{cm}$, proches de nos valeurs obtenues. Les eaux avec un aspect très trouble proviennent de puits peu profonds, ce qui explique leurs turbidités élevées. Les faibles valeurs de dureté des eaux relevées ne permettent pas l'élaboration de couche carbonatée pouvant participer à une protection des canalisations contre certains risques de corrosion (Rodier, 2009). De ce fait, une eau trop douce est agressive vis-à-vis des canalisations; en particulier la corrosion des canalisations en plomb devient dangereuse pour la santé du consommateur (Beauchamp, 2006). L'échantillon 10 par contre est une eau dure. Cette eau dure donne des dépôts de tartre dans les canalisations, les bouilloires et chauffe-eau, ainsi que dans les filtres des robinets. D'autre part, ces dépôts carbonatés ont un effet bénéfique en protégeant les conduites de la corrosion.

Les teneurs en cations majeurs: $\mathrm{Ca}^{2+}$, $\mathrm{Mg}^{2+}, \mathrm{Na}^{+}$et $\mathrm{K}^{+}$sont en dessous des normes 
OMS pour ces éléments. Les anions majeurs $\mathrm{HCO}_{3}^{-}, \quad \mathrm{Cl}^{-}, \mathrm{NO}_{3}^{-}$et $\mathrm{SO}_{4}{ }^{2-}$ présentent également des teneurs en dessous des normes OMS (UNE, 2007). Les bicarbonates sont les ions les plus dominants dans les échantillons d'eau et ces ions représentent plus de $50 \%$ de la charge anionique dissoute. Les bicarbonates constituent l'essentiel de l'alcalinité des eaux de cette localité. Ils peuvent provenir de la dissolution de formations carbonatées selon la réaction :

$$
\mathrm{CaCO}_{3}+\mathrm{H}_{2} \mathrm{O} \rightarrow 2 \mathrm{HCO}_{3}{ }^{-}+\mathrm{Ca}^{2+}
$$

Les faibles valeurs en nitrates obtenues indiquent que les eaux étudiées ne sont pas sujettes à un risque de pollution par les nitrates, mais ces valeurs indiquent des rejets d'eaux usées et surtout une utilisation excessive de fertilisants agricoles.

Le fer est indispensable pour le corps humain, mais de très fortes concentrations affectent les caractéristiques organoleptiques de l'eau et tachent le linge. La présence de fer dans l'eau peut favoriser la prolifération de certaines souches de bactéries qui précipitent le fer où corrodent les canalisations. La présence du manganèse dans l'eau représente d'abord une nuisance organoleptique (goût métallique) et esthétique (couleur noire). Cette nuisance peut être ressentie par les consommateurs à la concentration de 0,03 $\mathrm{mg} / \mathrm{L}$ (Raveendran et al., 2001). Une surcharge de l'organisme humain en fer peut entraîner une hémochromatose primitive (mauvaise régulation de l'absorption du fer par l'intestin) et même une cancérisation hépatique (risque de cancer du foie). Le manganèse peut également être à l'origine de troubles neurologiques irréversibles à cause de son antagonisme vis-à-vis du calcium. Ces troubles interviennent généralement lorsque les concentrations en fer et en manganèse sont respectivement supérieures à $10 \mathrm{mg} / \mathrm{L}$ et 2 $\mathrm{mg} / \mathrm{L}$ (Lanciné et al., 2008). Les teneurs en fer (II) et en manganèse (II) dépassent les normes OMS, ceci justifie les plaintes des populations quant à la qualité organoleptique de ces eaux.
Le paysage géologique de la zone de Yamtenga est constitué d'un granite gris à grain moyen à biotite et rare en muscovite. Dans la composition usuelle du type de granite de notre zone d'étude, le fer et le manganèse ne sont pas des constituants majeurs. Une autre explication à la présence de ces éléments dans les eaux souterraines doit alors être trouvée. Une hypothèse serait que ces éléments proviendraient de l'infiltration des eaux du barrage. L'activité humaine avec la proximité de la ville contribue grandement à la pollution des eaux du barrage et de ses bras. Ces eaux dont les caractéristiques organoleptiques sont altérées font donc l'objet d'un rejet de la part des populations pour l'usage en eau de boisson, ce qui justifie leurs plaintes.

Le thallium se rencontre dans la nature sous forme d'impuretés dans les pyrites et les blendes. Il ne se trouve pas habituellement dans les eaux naturelles. Sa présence peut être due aux rejets des industries de fabrication de produits insecticides et raticides, de cellules photo-électriques, de lampes. Il est hautement toxique pour la biosphère. Sa toxicité étant même supérieure à celui du mercure, cadmium, plomb et cuivre (Spano et al., 2005). Les risques liés à cet élément imposent que les eaux avec les fortes teneurs en thallium enregistrées soient évitées comme eau de boisson.

Compte tenu de la charge ionique élevée de certaines eaux des nappes de l'agglomération de Ouagadougou, une erreur de $10 \%$ est acceptable sur la balance ionique. Une erreur sur la balance ionique peut provenir d'une erreur analytique (résultats des analyses non valides) ou d'une minéralisation particulière (ions présents dans l'eau qui n'ont pas été analysés) (Ouandaogo/Yaméogo, 2008). La plupart des eaux analysées ont une balance ionique inférieure à $10 \%$, confirmant ainsi que les résultats sont fiables. Cependant, lors de certaines campagnes et pour certains points d'eau (points 1, 6, 8), nous avons eu 
des balances ioniques supérieures à la valeur acceptable de $10 \%$. Ces eaux pourraient donc contenir d'autres éléments non-pris en compte pour le calcul de la balance ionique, notamment les éléments traces métalliques comme le fer et le manganèse qui sont élevés pour certains points d'eaux.

Les faciès hydrochimiques trouvés confirment les travaux de Ouandaogo/Yaméogo (2008) qui a travaillé sur les eaux du socle de Ouagadougou constitué essentiellement de roches granitoïdes, les eaux de forages étant caractérisées par un faciès bicarbonaté calcique et magnésien. Oga et al. (2009) ont travaillé sur les eaux des aquifères de fracture de la région de Tiassalé (Côte d'ivoire). Leurs travaux ont montré que le paysage géologique de cette région est constitué de granitoïdes éburnéens et l'alimentation en eau potable se fait au moyen de forages captant le plus souvent les aquifères fissurés. Les eaux de ces forages sont à majorité d'un faciès bicarbonaté calcique et magnésien et la plupart de ces eaux indiquent des fortes teneurs en fer et en manganèse.

\section{Conclusion}

Dans ce travail, nous avons analysé les paramètres physico-chimiques des eaux souterraines utilisées comme source d'eau potable par les populations rurales du village de Yamtenga en vue d'évaluer la qualité de ces eaux. Cette étude a montré que ces eaux souterraines ne sont pas conseillées à la consommation comme eau de boisson. Les paramètres qui déclassent ces eaux souterraines comme eau de boisson sont le fer, le manganèse et le thallium pour certains points d'eau. Il y a donc lieu de procéder à une purification de ces eaux en vue de l'élimination de certains polluants métalliques que sont $\mathrm{Fe}^{2+}, \mathrm{Mn}^{2+}$ et $\mathrm{Tl}^{+}$.

Les cations majeurs présentent un ordre d'abondance du type : $\mathrm{Ca}^{2+}>\mathrm{Mg}^{2+}>$ $\mathrm{Na}^{+}>\mathrm{K}^{+}$
Les anions majeurs se présentent dans l'ordre suivant: $\mathrm{HCO}_{3}{ }^{-}>\mathrm{Cl}^{-}>\mathrm{SO}_{4}{ }^{2-}>\mathrm{NO}_{3}{ }^{-}$

Les eaux souterraines de Yamtenga sont classées bicarbonatées calciques et magnésiennes pour la totalité des ouvrages échantillonnés avec un début d'évolution du pôle bicarbonaté vers le pôle chlorures + nitrates, traduisant ainsi des apports de surface (pollution anthropique).

\section{REMERCIEMENTS}

Ce travail a été financé par «International Science Programme (ISP/IPICS), Uppsala, Sweden». Les auteurs expriment leurs remerciements pour l'appui matériel et financier dont ce travail a bénéficié.

\section{REFERENCES}

Beauchamp J. 2006. Qualité et Pollution des Eaux Souterraines. Université de Picardie Jules-Verne.

Belghiti ML, Chahlaoui A, Bengoumi D. 2013. Caractéristiques physico-chimiques des eaux de certains puits utilisés comme source d'eau potable en milieu rural dans la région de Meknes (MAROC). ScienceLib Editions Mersenne, 5: 1-16.

Bouchemal F, Bouchahm N, Achour S. 2011. Qualité des eaux des nappes aquifères de la région de BISKRA. Revue des Sciences Fondamentales et Appliquées, 3(1): 3444.

Derwich E, Benaabidate L, Zian A, Sadki O, Belghity D. 2010. Caractérisation physico-chimique des eaux de la nappe alluviale du Haut Sebou en aval de sa confluence avec OUED FES. Larhyss Journal, 8: 101-112.

El Asslouj J, Kholtei S, El Amrani-Paaza N, Hilali A. 2007. Impact des activités anthropiques sur la qualité des eaux souterraines de la communauté Mzamza (Chaouia, Maroc). Revue des Sciences de l'Eau, 20(3): 309-321. 
Kombassere A. 2007. L'accès à l'eau potable et les risques diarrhéiques dans les zones irrégulières de Ouagadougou : les cas de Yamtenga. Mémoire de Maîtrise de Géographie, Université de Ouagadougou.

Kouassi AM, Mamadou A, Ahoussi KE, Biemi J. 2013. Simulation de la conductivité électrique des eaux souterraines en relation avec leurs propriétés géologiques : cas de la Côte d'Ivoire. Rev. Ivoir. Sci. Technol., 21-22: 138-166.

Lanciné GD, Touchard AJ, Bamory K, Fernand K, Kouadio K, Issiaka S. 2008. Elimination du fer et du manganèse par aération-filtration des eaux de forage en zone rurale dans les pays en développement, cas de la région de Tassialé (Sud de la cote d'ivoire), European Journal of Scientific Research, 19(3): 558-567.

Matini L, Moutou JM, Kongo-Mantono MS. 2009. Evaluation hydro-chimique des eaux souterraines en milieu urbain au Sud-ouest de Brazzavile, Congo. Afrique Science, 5(1): 82-98.

OEHHA (Office of Environmental Health Hazard Assessment). 1999. Public health goal for thallium in drinking water. California Environmental Protection Agency.

Oga M-S, Lasm T, Yao TK, Soro N, Saley MB, Kouassi D, Gnamba F. 2009. Caractérisation chimique des eaux des aquifères de fracture : cas de la région de Tiassalé en Côte D'ivoire. European Journal of Scientific Research, 31(01): 72-87.

Ouandaogo/Yaméogo S. 2008. Ressources en eau souterraine du centre urbain de Ouagadougou au Burkina Faso, qualité et vulnérabilité. Thèse de doctorat, Université d'Avignon et des Pays de Vaucluse.

Raveendran R, Ashworth B, Chatelier B. 2001. Manganese removal in drinking water système, $64^{\text {th }}$ annual Water Industry Engineers and Operator's Conference, Bendigo.

Rodier J. 2009. L'Analyse de l'Eau (9ème édition). Ed. Dunod : Paris.

Simler R. 2007. Diagramme 5.6. Logiciel Libre du Laboratoire d'Hydrogéologie. Université d'Avignon, France.

Spano N, Panzanelli A, Piu PC, Pilo MI, Sanna G, Seeber R, Tapparo A. 2005. Anodic stripping voltammetric determination of traces and ultratraces of thallium at a graphite microelectrode, method development and application to environmental waters. Analytica Chimica Acta, 553: 201-207.

UNE (United Nation Environment). 2007. Program Global Environment Monitoring System, Global drinking water quality index development and sensitivity analysis report. UNE. 